\title{
Catalytic flash gasification of EFB for hydrogen production using zeolite supported metal oxide catalysts
}

\begin{abstract}
Empty fruit bunch (EFB) waste is produced in large amount in Malaysia from intense oil palm agriculture activity. Direct usage of EFB as a source of energy is not economically feasible and ideally should be upgraded before it can produce green energy economically. Current gasification processes produces a lot of tar while yielding low amount of hydrogen. Flash gasification of EFB with the presence of catalysts shows improvements over the uncatalysed reaction. In this study, by using a high surface area support catalyst of ZSM-5 with the presence of $1 \% \mathrm{Ni}, \mathrm{Zn}$, or Fe metal loading is sufficient to enhance the hydrogen production. ZSM-5, NiO/ZSM-5, CuO/ZSM-5, Fe2O3/ZSM-5 and ZnO/ZSM-5 catalyst with 1 wt \% loading were prepared via the wet impregnation method. XRD patterns of the prepared catalysts shows almost identical peaks patterns which indicates high dispersion of dopants on the support catalyst Flash gasification was carried out at $900^{\circ} \mathrm{C}$ under isothermal heating conditions with $10 \mathrm{sccm} 4.99 \%$ O2 diluted in He. Syngas produced was then analysed using an online quadrupole mass spectrometer. Catalytic activity for hydrogen production is the highest for NiO/ZSM-5 followed by ZnO/ZSM-5, Fe2O3/ZSM-5, ZSM-5.
\end{abstract}

Keyword: Catalysis; EFB; Gasification; Hydrogen; Tar cracking reaction 